\title{
Nonperturbative treatment of medium-energy proton scattering under shadowing-blocking conditions in $\mathrm{Al}(110)$
}

\author{
P. L. Grande and A. Hentz \\ Instituto de Física da Universidade Federal do Rio Grande do Sul, Avenida Bento Gonçalves 9500, 91501-970, Porto Alegre, RS, Brazil \\ G. Schiwietz \\ Hahn-Meitner-Institut, Abteilung SF4, Glienicker Strasse 100, 14109 Berlin, Germany
}

W. H. Schulte, ${ }^{*}$ B. W. Busch, ${ }^{\dagger}$ D. Starodub, and T. Gustafsson

Department of Physics and Astronomy, and Laboratory for Surface Modification, Rutgers University, 136 Frelinghuysen Road, Piscataway, New Jersey 08854-8019, USA

(Received 5 August 2003; published 29 March 2004)

\begin{abstract}
Measurements of the energy spectrum for $98 \mathrm{keV}$ protons backscattered from $\mathrm{Al}(110)$ under shadowingblocking conditions have been performed with high resolution. The corresponding energy losses at central collisions are dominated by ionization of the Al inner shells. In connection with coupled-channel calculations for the electronic energy loss in individual atomic collisions, we discuss the influence of higher-order effects and surface relaxation in the simulation of the strongly asymmetric surface peak.
\end{abstract}

DOI: 10.1103/PhysRevB.69.104112 PACS number(s): 61.85.+p, 34.50.Bw, 34.50.Fa, 79.20.Rf

\section{INTRODUCTION}

The energy loss of energetic ions in matter has been studied extensively over several decades, not only aiming a better understanding of the basic processes ${ }^{1}$ but predominantly because of its relevance for ion beam analysis and materials modification.

Medium-energy ion scattering (MEIS) in connection with shadowing and blocking techniques is a powerful method for the determination of structural and vibrational parameters of crystalline surfaces. ${ }^{2}$ However, the shape of ion energy-loss spectra is usually not analyzed, because this requires a detailed knowledge on the energy-transfer mechanisms. The differential excitation/ionization probability for each subshell in a single collision is the important quantity in this case, since generally only few collisions are involved. Thus, standard energy-loss theories or semiempirical methods based on Gaussian energy-loss distributions cannot be used successfully. Instead, an atomistic description of the electronic excitation process and its impact parameter dependence have to be taken into account in a stochastic approach which leads, in general, to an asymmetric line shape.

In our high-resolution ion scattering experiments, we have used a channeling and blocking configuration of the ion beam as well as the detector and a single crystal as the target. By using channeling, we ensure that only the first few atoms on each atomic string will be hit by the incoming ion beam. By positioning the detector in a blocking configuration, i.e., along a high symmetry direction of the target, the detected signal from lower-lying layers will be further suppressed. The spectrum will have a narrow leading peak, the surface peak. As only a few surface layers have to be considered, the computation of the line shape of this peak is simplified. As shadowing and blocking are present and the projectile scattering angle is large, only collisions with very small impact parameters are important. Since solid-state effects are of minor importance due to the large energy transfers involved, the valence-band contributions to the backscattering signal may therefore to a good approximation be described within an atomic model. This provides the best scenario for the use of advanced atomic-physics models, such as coupled-channel calculations.

Here we report on measurements and corresponding simulation of the energy-loss distribution of the surface peak for protons impinging with $60^{\circ}$ (and $\phi=35.3^{\circ}$ ) with respect with the main axes of a clean $\mathrm{Al}(110)$ surface and being backscattered along a blocking direction also $60^{\circ}$ off normal. We provide the first full $a b$ initio simulation of the surface peak, a structure that has been widely measured in channeling experiments, but the detailed shape of which has never been analyzed.

\section{EXPERIMENTAL PROCEDURE}

The surface peak is due to collisions at and near the surface, involving just the first few atomic layers. The deflection of the incoming projectiles by surface atoms results in the formation of a volume behind this atom, practically free of ion trajectories, the so-called shadow cone. If the incident ion beam is aligned with a main axis of the crystal, shadowing greatly reduces the chance of backscattering from successive atoms along the row. In a similar way, the backscattered flux from subsurface atoms cannot propagate in directions corresponding to vectors that point to atoms closer to the surface. This will result in pronounced minima in the angular distribution of the backscattered flux. Such blocking dips provide a sensitive method to determine surface-atom displacements. An angular shift in the position of a blocking dip away from the bulk crystal blocking direction is a direct indication of layer relaxation. Accurate determination of structural parameters (atomic location and vibrational amplitudes) using MEIS is a well-established technique. This is accomplished by comparing the angular scattering intensity to results of Monte Carlo type computer simulations for 


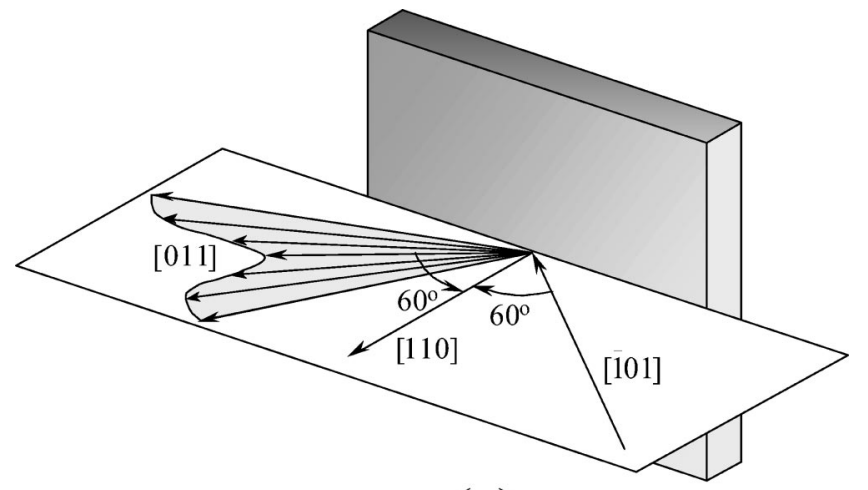

(a)

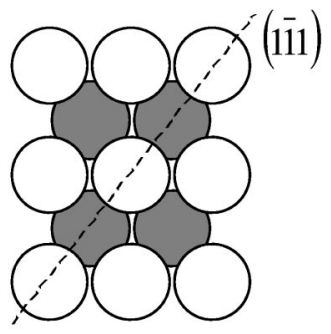

(b)

FIG. 1. (a) Schematic figure of the scattering geometry used in this work; the scattering plane is shown in (b) in a top view of the $\mathrm{Al}(110)$ surface.

models of the surface structure, as implemented, e.g., in the VEGAS (Ref. 3) code for trial atomic crystal structures. The atomic scattering cross sections are well known for the energy range $(100 \mathrm{keV})$ in question. The atomic positions in the trial structure are changed until a convincing fit to the measured data (shape and minimum position) is obtained. This method, however, takes into account only the ballistic part of the backscattering events. The information contained in the detailed peak shape is usually not considered. The scattering experiments have been performed in a UHV chamber with a base pressure of $1 \times 10^{-10}$ Torr. $98-\mathrm{keV}$ protons are directed into the chamber from a $400 \mathrm{keV}$ Ion Implanter, manufactured by High Voltage Engineering Europe B. V. The ions, scattered on the target, are dispersed in energy in a highresolution toroidal electrostatic analyzer. ${ }^{4} \mathrm{~A}$ two-dimensional (2D) position sensitive charge-dividing collector in conjunction with microchannel plates ${ }^{5}$ provides simultaneous detection of ions in a range of energies and scattering angles. The auxiliary equipment includes a low-energy electron diffraction (LEED) system for monitoring surface orientation and structure. The $\mathrm{Al}(110)$ crystal was electropolished in a solution of perchloric acid and acetic anhydride. After mounting into the UHV chamber, it was cleaned by several cycles of sputtering with $1.0-\mathrm{keV} \mathrm{Ne}^{+}$ions and sequential annealing at $720 \mathrm{~K}$, until a sharp $(1 \times 1)$ LEED pattern could be observed. No carbon and oxygen contamination could be detected by MEIS (sensitivity of about 0.05 monolayer) during data acquisition. The configuration of the scattering experiment is schematically sketched in Fig. 1(a). The top view of the $\mathrm{Al}(110)$ surface with a scattering plane perpendicular to
TABLE I. The table contains the following items for each layer $L$ of the $\mathrm{Al}(110)$ surface: combined hitting and detecting $p_{H D}$ as well as hitting $p_{H}$ probabilities according to the VEGAS program, hitting probabilities $p_{H}$ obtained from Appendix A, 1D thermal vibrations, the nuclear energy-loss broadening obtained from Appen$\operatorname{dix} \mathrm{B}$, and the experimentally determined surface relaxation in terms of the bulk interlayer separation.

\begin{tabular}{lcccccc}
\hline \hline $\begin{array}{l}\text { Layer } \\
(L)\end{array}$ & $\begin{array}{c}p_{H D} \\
\text { VEGAS }\end{array}$ & $\begin{array}{c}p_{H} \\
\text { VEGAS }\end{array}$ & $\begin{array}{c}p_{H} \\
\text { Appendix A }\end{array}$ & $\begin{array}{c}\sigma_{\text {thermal }} \\
(\AA)\end{array}$ & $\begin{array}{c}\sqrt{\delta E^{2}} \\
(\mathrm{eV})\end{array}$ & $\begin{array}{c}\text { Relaxation } \\
(\%)\end{array}$ \\
\hline 1 & 0.989 & 0.990 & 1 & 0.164 & 0 & -8.5 \\
2 & 0.878 & 0.945 & 0.935 & 0.136 & 62.1 & +4.9 \\
3 & 0.549 & 0.730 & 0.726 & 0.113 & 87.7 & -2.5 \\
4 & 0.253 & 0.459 & 0.461 & 0.107 & 105.4 & 0 \\
5 & 0.086 & 0.241 & 0.231 & 0.105 & 116.6 & 0 \\
6 & 0.031 & 0.108 & 0.091 & 0.104 & 124.5 & 0 \\
7 & 0.008 & 0.047 & 0.029 & 0.104 & 130.3 & 0 \\
\hline \hline
\end{tabular}

the $\mathrm{Al}(110)$ surface is given in Fig. 1(b). The incoming beam is aligned with the [101] direction. The energy spectra of the scattered ions are simultaneously collected in an angular range of $20^{\circ}$, centered about the scattering angle $60^{\circ}$. For a bulk-terminated static lattice this geometry completely eliminates scattering events from all layers except the topmost one. Due to thermal vibrations and surface relaxation the deeper layers contribute to the surface peak as well. To obtain the absolute $\mathrm{Al}$ yield, we have used Rutherford scattering cross sections with a simple angle-independent correction for the electronic screening in the effective kineticenergy formulation. ${ }^{6}$ The neutralized fraction of the scattered ions, which is not available for electrostatic analysis, was measured using a surface barrier detector. It constituted $11 \%$ for ions with incident energy of $98 \mathrm{keV}$. The angular distribution of the surface peak yield was fitted using the VEGAS simulation. The best fit values of near-surface atom relaxation and vibrational amplitudes are summarized in Table I. For better statistics, the energy spectra have been summed up in an angular range of $2^{\circ}$ about the blocking minimum position to produce the surface peak analyzed below.

\section{THEORETICAL PROCEDURE}

In order to describe the shape of the surface peak, we may subdivide the slowing down process of the ions into two parts. The first step consists in the determination of the probability for the different energy losses in single atomic collisions with the relevant impact parameters. In the second step the sequence of collisions is treated stochastically by convolution of the energy-loss distributions for all atoms along the ion path. The corresponding backscattering and detection probabilities that define the ion histories are treated analytically in Appendix A. These hitting and detecting probabilities have been used in the present work and they are nearly identical to those determined by the well-known Monte Carlo simulation program VEGAS. ${ }^{3}$

\section{A. Single collisions}

Coupled-channel calculations are the best tool to describe inner-shell ionization and excitation of atoms ${ }^{7,8}$ as a function 
of the impact parameter. These time consuming calculations are based on the semiclassical method. ${ }^{9}$ The projectile following a classical trajectory provides a time-dependent electrostatic perturbation on the target electrons. Hence, the timedependent Schrödinger equation is solved by expanding the electronic wave function in a truncated basis of states, namely atomic orbitals. A set of first-order ordinary coupled differential equations for the coefficients originating from this expansion, the so-called coupled-channel equations, is integrated numerically along the classical trajectory of the projectile for a given impact parameter $b$. Thus, the amplitude $a_{i \rightarrow f}$ is calculated for a given transition from an initial occupied state $i$ to an unoccupied bound or continuum state $f$ and thus the probability corresponding to atomic excitation or ionization is determined. For bare incident ions, the active-electron interaction is just the Coulomb potential. However, screening of the projectile charge can be easily incorporated in the calculation. Details of the atomic orbital coupled-channel calculations (AO) may be found elsewhere. ${ }^{8}$ Furthermore, for the present case coupled-channel calculations are found to be superior over simple ones based on first-order perturbation theory. ${ }^{10}$

The independent-electron model (IEM) is adopted for one active electron in the target atom moving in the electrostatic field due to both nuclei and the other electrons, which are included in a frozen-core Hartree-Fock-Slater framework. ${ }^{11}$ In this way, the ground-state and excited-state wave functions (where the hole in the $i$ th shell is not accounted for in the self-consistent potential) as well as the energies of the active electron are calculated. Since each of the 400 excited or continuum state corresponds to a well-defined energy transfer $T=\varepsilon_{f}-\varepsilon_{i}$, the electronic energy-loss probability is given by

$$
\frac{d P_{i}}{d T}(b)=\sum_{f}\left|a_{i \rightarrow f}(b)\right|^{2} \delta\left(T-\left(\epsilon_{f}-\epsilon_{i}\right)\right),
$$

where the sum above means an integral over $\epsilon_{f}$, in the case of continuum states. It is pointed out that for elastic collisions $(f=i)$ as well as for "bound-state" excitation the energy-loss distribution defined above contains spikes due to the atomic level structure. Broadening effects originating from state lifetime, bandwidth, and the Doppler effect are not considered explicitly since they are much smaller than the experimental resolution.

In the framework of the independent-electron model, the probability for a certain total electronic energy-loss $\Delta E$ transferred during an individual ion-atom collision can be written as

$$
\frac{d P_{\text {atom }}^{\text {elec }}}{d \Delta E}(b)=\left(\prod_{i} \int d T_{i} \frac{d P_{i}}{d T_{i}}(b)\right) \times \delta\left(\Delta E-\sum_{i} T_{i}\right)
$$

where the index $i$ runs over all electrons for each subshell $1 s, 2 s, 2 p, 3 s$, and $3 p$ of the $\mathrm{Al}$ atom. Equation (2) corresponds to a series of convolutions of individual singleelectron energy-loss distributions.

Figure 2 shows the results of the coupled-channel calcu-

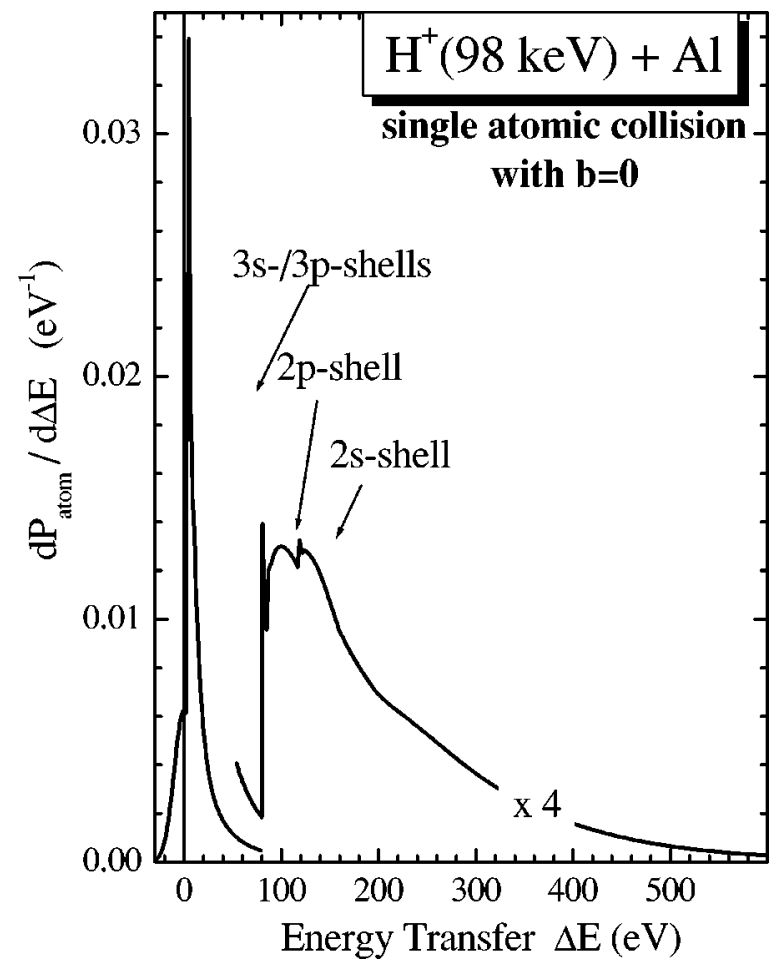

FIG. 2. Energy transfer in a single collision for $98 \mathrm{keV} \mathrm{H}^{+}$on atomic $\mathrm{Al}$ at an impact parameter of $b=0$.

lations for the energy-loss probability of $98 \mathrm{keV} \mathrm{H}^{+}$projectiles colliding with atomic $\mathrm{Al}$ at $b=0$. The elastic peak (the peak at $\Delta E=0$ ) is represented here by a Gaussian distribution with a very small standard deviation (about $10 \mathrm{eV}$ ) in order to keep the normalization and to avoid the use of a Dirac function. We would like to point out that in contrast to approaches based on perturbation theory, all results from the coupled-channel method are unitary (occupation probabilities sum up to one for each active electron). This unitary behavior leads to a reduction of the elastic scattering intensity when inelastic channels are important. In the present case, backscattering is almost always accompanied by excitation or ionization events, involving mainly electrons from the $2 p$ shell and from the $3 s$ and $3 p$ bands. This is the reason for high backscattering yields at nonzero energies in the figure.

The main feature of the energy-loss distribution in Fig. 2 is the significant contribution of the $L$-shell at large energy transfers. The contribution of the valence electrons for the surface peak is of minor importance since the corresponding $M$-shell energy loss is much smaller than the experimental resolution. $K$-shell ionization of $\mathrm{Al}$ atoms is kinematically suppressed for protons at incident energies below about 1 $\mathrm{MeV}$.

Finally the nuclear energy loss (quasielastic atomic energy transfer) can be incorporated into Eq. (2) by assuming that the electronic and nuclear energy distributions are uncorrelated according to

$$
\frac{d P_{\text {atom }}}{d \Delta E}(b)=\frac{d P_{\text {atom }}^{\text {elec }}}{d \Delta E_{\text {elec }}}(b) * \frac{d P_{\text {atom }}^{\text {nucl }}}{d \Delta E_{\text {nucl }}}(b) .
$$


where the symbol * stands for a convolution of impact parameter dependent inelastic and elastic scattering probabilities. Usually the nuclear energy loss can be simply added to $\Delta E_{\text {elec }}$ since there is only one nuclear energy transfer at a given impact parameter. Test calculations accounting for the shadow cone show that this broadening effect (due to nuclear energy-loss straggling) is very small in the present case.

\section{B. Stochastic approach}

The energy lost by the projectile after several collisions is given by a series of convolutions of the energy-loss distribution for each single collision. Here a collision is defined as an ion penetration of a target Wigner-Seitz cell. This technical definition may also be extended to account for the energy loss due to the other surrounding target atoms, but these have been neglected in the present work. Thus, each single collision is characterized by one impact parameter $b_{\alpha}$. When a sequence of collisions is described by a set of impact parameters $\left\{b_{\alpha}\right\}$, for example, along the incoming path before hitting the target nucleus, the energy-loss distribution of the projectile will be

$$
\begin{aligned}
\frac{d P_{\text {in }}}{d \Delta E}\left(\left\{b_{\alpha}\right\}\right)= & \left(\prod_{\alpha} \int d \Delta E_{\alpha} \frac{d P_{\text {atom }}}{d \Delta E_{\alpha}}\left(b_{\alpha}\right)\right) \\
& \times \delta\left(\Delta E-\sum_{\alpha} \Delta E_{\alpha}\right)
\end{aligned}
$$

The measurable sequences of impact parameters $\left\{b_{\alpha}\right\}$ are however only those that lead to a hitting event (a close backscattering encounter) and will depend on shadowing effects due to the atomic layers, which the ions pass through (see Appendix A). In this way, the thermal vibrations as well as the relaxation of the first layers must also be taken into account to determine the impact-parameter-averaged energy loss due to a target atom from the layer $L^{\prime}$ for the projectile that hits a target atom from layer $L$,

$$
\left(\frac{d P_{\text {atom }}}{d \Delta E}\right)_{L^{\prime}, L}^{a v}=\frac{\int d^{2} b \frac{d P_{\text {atom }}}{d \Delta E}(b) g\left(\vec{r}_{\perp}-\vec{r}_{0 \perp}, \sigma\right)}{\int d^{2} b g\left(\vec{r}_{\perp}-\vec{r}_{0 \perp}, \sigma\right)}
$$

where the function $g$ is an integrated Gaussian distribution with standard deviation $\sigma$ described in the Appendix A. The impact parameter $b$ and $r_{\perp}(b)$ are the transverse positions of the impinging ion before and after the shadow cone (see Appendix A for details). The parameters $\vec{r}_{0 \perp}$ are offset parameters and will depend on surface relaxation depicted in Table I. Thus, we have replaced an explicit consideration of the Monte Carlo result for the collision sequences $\left\{b_{\alpha}\right\}$ and $\left\{b_{\beta}\right\}$ by ion histories that are described by straight lines for the incoming and outgoing ion paths. Nevertheless, as depicted in Table I, the hitting probability obtained using this assumption (see Appendix A) is very close to the one obtained from the Monte Carlo VEGAS program. ${ }^{3}$
The same holds true for the outgoing path after the backscattering. Then, the projectile energy distribution for a sequence of collisions that hit a target atom from layer $L$ and reach the detector reads

$$
\begin{aligned}
Y_{L}(E)= & \prod_{L^{\prime}=1}^{L} \int d \Delta E_{L^{\prime}}^{\text {in }}\left(\frac{d P_{\text {atom }}}{d \Delta E_{L^{\prime}}^{\text {in }}}\right)_{L^{\prime}, L}^{a v} \\
& \times \int d \Delta E^{h i t}\left(\frac{d P_{\text {atom }}}{d \Delta E^{\text {hit }}}(b=0)\right) \\
& \times \prod_{L^{\prime}=1}^{L} \int d \Delta E_{L^{\prime}}^{\text {out }}\left(\frac{d P_{\text {atom }}}{d \Delta E_{L^{\prime}}^{\text {out }}}\right)_{L^{\prime}, L}^{a v} \\
& \times \delta\left(E-\left[K\left(E_{0}-\sum_{L^{\prime}} \Delta E_{L^{\prime}}^{\text {in }}-\frac{1}{2} \Delta E^{h i t}\right)\right.\right. \\
& \left.\left.-\sum_{L^{\prime}} \Delta E_{L^{\prime}}^{\text {out }}-\frac{1}{2} \Delta E^{h i t}\right]\right),
\end{aligned}
$$

which turns out to be a series of convolutions. Here $E_{0}$ is the incident projectile energy and $K$ is the kinematical factor describing the two-body kinematics for the backscattering collision. ${ }^{12}$ All impact parameters involved in the equations above are small compared to the $L$ - and $M$-shell radii (they are given by the thermal vibrations of about $0.1 \AA$ ). We have assumed a straight-line motion for the incoming and outgoing ion path for the averaging of the energy loss over the impact parameter. In fact the angular deviations affect the kinematical factor and have also been taken into account (see Appendix B) in this investigation. They are relevant mainly for heavier ions at lower energies.

All above energy distributions are by definition normalized to one. The measurable projectile energy-loss function can be determined by (neglecting the energy resolution of the detection system)

$$
Y_{\text {detected }}(E)=\sum_{L} p_{H D}(L) Y_{L}(E)
$$

where $p_{H D}$ is the hitting-detecting probability for a backscattering collision at a given layer $L$. The hitting probability $p_{H}$ is the chance to hit a certain target atom in a close backscattering encounter and will depend basically on shadowing effects due the layers the ions pass through. The detecting probability $p_{D}$ is the chance for a particle emerging from that target position to leave the crystal in the detector direction and will depend strongly on blocking. For the upper layers $p_{H D}$ is very close to the product of hitting $p_{H}$ and detecting $p_{D}$ probabilities as observed also previously. ${ }^{3}$ Thus, for layers $L<4$ the effect of connected in and out tracks is minor.

For the 60-60 degree geometry in $\mathrm{Al}(110)$, the number of collisions for the backscattering occurring in layer $i$ (incom- 


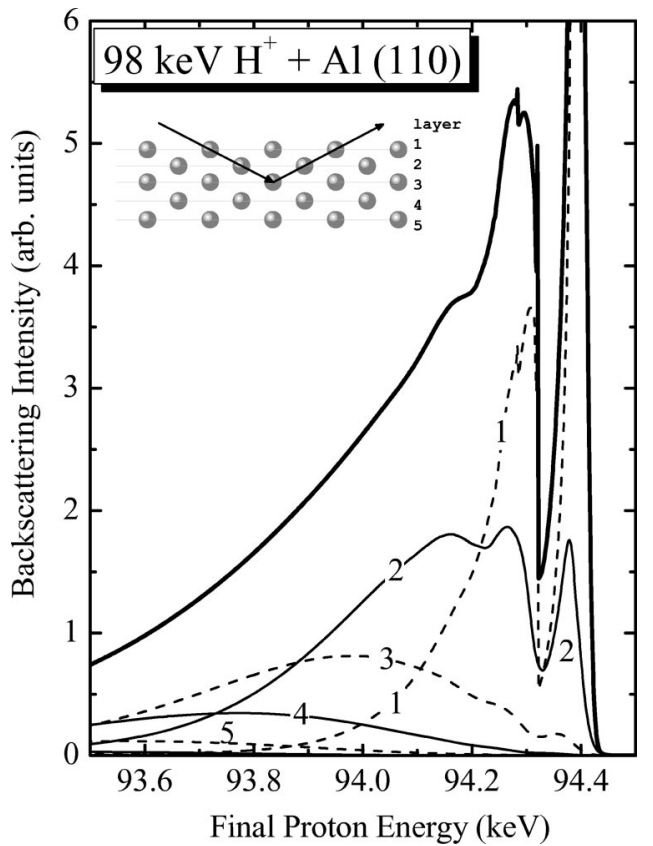

FIG. 3. Contribution of each visible layer for the surface peak of $98 \mathrm{keV} \mathrm{H}^{+}$backscattered from the $\mathrm{Al}(110)$ surface for a 60-60 degree geometry (see inset and also Fig. 1).

ing and outgoing along the closed packed $\langle 111\rangle$ direction) is $2 i-1$. The contributions of each of the layers are very accurately given by the hitting-detection probabilities $\left(p_{H D}\right)$ from the VEGAS code $^{3}$ with the experimental structural parameters $^{13,14}$ (see Table I).

The energy-loss distribution for a fixed impact-parameter $b=0$ of each visible backscattering layer is displayed in Fig. 3. The contribution of the first layer corresponds to a single collision with the first atom on the Al surface (see Fig. 2) (here it is plotted as a function of the backscattered ion energy). The deeper layers involve differences in the impactparameter distribution and a convolution of these layerspecific distributions [see Eq. (6)]. For backscattering layers $L>3$ the resulting shape is approaching a Gaussian distribution, as expected for electronic energy losses. For these deep layers, the shape of the energy-loss spectrum may be described by two parameters, namely, a mean energy loss (104 eV/layer for a single ion passage) and a width related to the so-called electronic straggling.

As for surface vibrations, a procedure similar to that used to obtain the dynamic parameters ${ }^{14}$ has been used. The bulk 1D rms amplitude is $0.104 \AA$ [corresponding to a Debye temperature of $390 \mathrm{~K}$ (Ref. 15)]. The surface layer has a relatively large amplitude enhancement (more than 50\%), consistent with it being an open surface. The second and third layers also have enhancements of about $25 \%$ and $10 \%$, respectively. These values have been obtained from the absolute MEIS yield.

According to Eq. (7) the area of each curve in Fig. 3 corresponds to the hitting-detecting probabilities $p_{H D}$. The backscattering yield of the first and second layers are similar because of a comparatively large surface relaxation, leading to a layer offset of about $0.1 \AA$ (see Table I). The third and

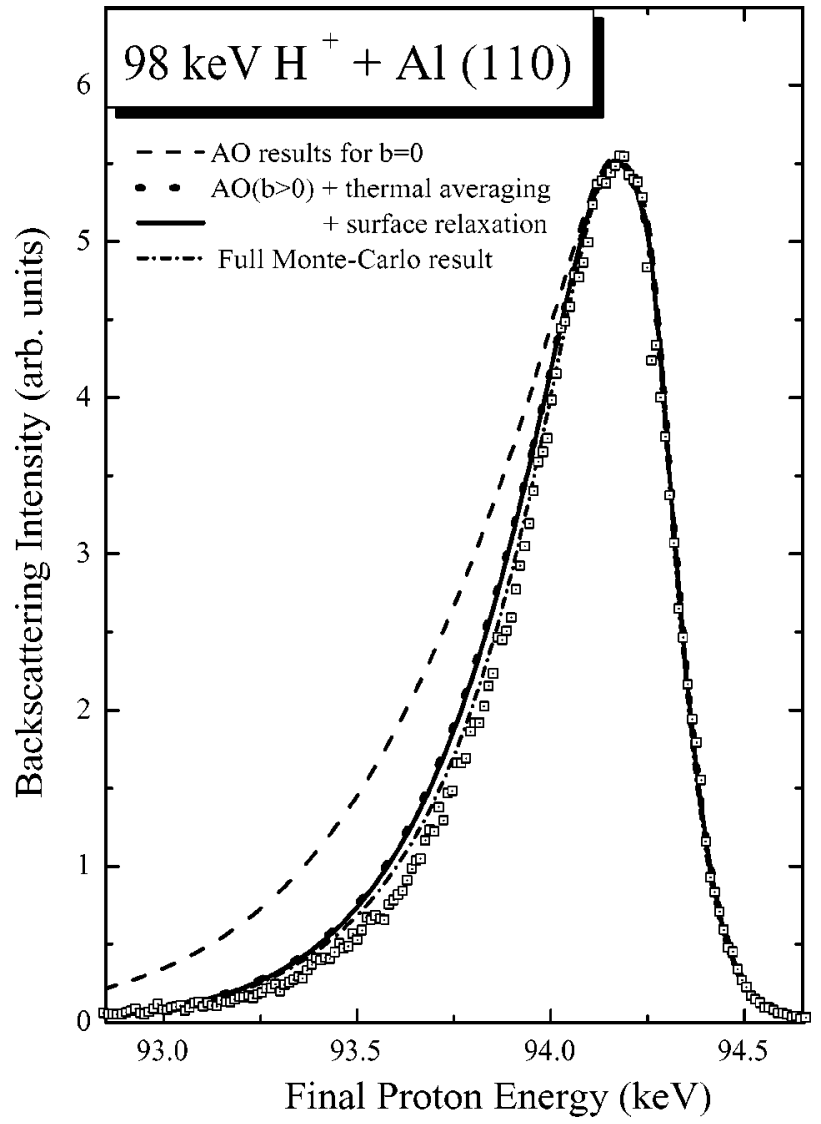

FIG. 4. Experimental data (open squares) for $98 \mathrm{keV} \mathrm{H}^{+}$backscattered from $\mathrm{Al}(110)$ in comparison with simulations using the coupled-channel method (AO), the hitting-detecting probabilities from Table I, impact-parameter average according Appendix A and the broadening from Appendix B. Dashed line: energy-loss calculations for $b=0$ only [Eq. (5) is replaced by the zero impactparameter energy-loss distribution], weighted with the hittingdetecting probabilities. Dotted line: energy-loss calculations using Eqs. (1)-(7), averaged over thermal vibrations and considering the weighted impact-parameter dependence of the energy-transfer distributions. Solid curve: energy-loss calculations including thermal vibrations and additionally the Al surface relaxation. Dash-dotted curve: full Monte Carlo calculations (Ref. 16) including thermal vibrations and surface relaxation. All theoretical results have been convoluted with the spectrometer function and with the broadening form Appendix B.

fourth layers are still visible because of the thermal vibrations that are of the order of the shadow cone radius for the ion energy used. For large energy losses even the fifth layer gains some importance. However, layers $L>7$ have been neglected in the present work, since their contribution to the spectrum is very small.

\section{DISCUSSION}

In Fig. 4 we show the experimental energy distribution of $98 \mathrm{keV}$ incident protons backscattered from clean $\mathrm{Al}(110)$ compared with simulations using the coupled-channel method described above. All simulated theoretical spectra in the figure have been convoluted with the experimental reso- 
lution function. From various experiments it is clear that the relative spectrometer resolution is significantly below $0.2 \%$, dependent mainly on the beam-spot size. Thus, a realistic spectrometer function can only be determined from a backscattering experiment using a well-collimated beam. In this work we have convoluted our final theoretical results with a spectrometer function $S$ of the following type:

$$
S=\exp \left(-\ln (2)\left|2\left(E-E_{0}\right) / w_{S}\right|^{x_{S}}\right) .
$$

This function, convoluted with the theoretical spectrum, was fitted to the experimental data at the right-hand side of the surface backscattering peak. Such a procedure involves only a minor influence on the theoretical energy distribution, since elastical scattering from $\mathrm{Al}$ and conduction-band energy losses may roughly be approximated by a common $\delta$ function. The fit yields an exponent of $x_{S}=1.5$ (sharper peak, but extended wings compared to a Gaussian function) and a full width at half maximum of $w_{S}=180 \mathrm{eV}$ for the present experimental conditions.

The calculated curves in Fig. 4 have been convoluted with the energy distribution of Eq. (8) using exactly the same shape parameters. The dashed curve AO (atomic orbital coupled-channel calculations) corresponds the simulations according to Eq. (7) using a fixed impact parameter of $b$ $=0$ for the calculation of excitation/ionization probabilities in Al. The effect of the thermal vibrations along the ion history is represented by the dotted curve. Hence, the possible impact-parameters due to thermal vibrations are restricted to those outside of the shadow cone for a given hitting as well as detecting event. The solid curve includes additionally the effect of surface relaxation described in Table I. It should be stressed that all these effects have been already included in the hitting-detecting probabilities as obtained by backscattering-intensity simulations for this surface, ${ }^{14}$ but here they are considered for the first time in a computation of the backscattering energy spectrum.

The results of the simulation show that although the surface relaxation is very important for the total yield, it affects the shape of energy-loss distribution very little. This is because backscattering collisions taking place in deep layers that can contribute to the surface peak are due to trajectories having large impact parameters in the first layers (resulting in relatively insignificant energy losses) because of shadowing for the incoming path and blocking for the outgoing path. Thus, the surface relaxation plays a minor role for the energy-loss shape, but is very important for the total yield.

The simulation represented by the solid curve includes all important terms that can be computed within the framework of the IEM. In this simulation the effects concerning the electronic and atomic structures of $\mathrm{Al}(110)$ and ion-atom collisions including all higher-order effects have been very accurately included (within the IEM). Other effects such as a better description of the valence electrons, dynamically curved ion trajectories and/or the influence of a small $\mathrm{H}^{0}$ charge-state fraction are of minor importance. This has been checked using test calculations. Furthermore, previous investigations have shown that correlated vibrations only have a minor influence on the results. ${ }^{3}$ Also the approximations used to calculate the impact-parameter distributions for each collision as well as the angular spreading (described in Appendixes $\mathrm{A}$ and $\mathrm{B}$ ) have been cross checked by performing full Monte Carlo calculations. ${ }^{16}$ As can be observed in Fig. 4, there is reasonable agreement between the present analytical simulation (solid line) and a full Monte Carlo calculation for the same problem (dash-dotted curve). The maximum relative deviation between both model results slightly exceeds $10 \%$ at backscattering energies below $93.8 \mathrm{keV}$. This deviation might be an indication for the influence of remaining spatial correlations between incoming and outgoing paths or of small deviations from the straight-line assumption in Appendix A.

The experimental data show a steeper decrease towards lower energies than our best analytical prediction (solid line) or the Monte Carlo result (dashed-dotted curve). The remaining difference between both calculations and the experimental data clearly exceeds $30 \%$ at low backscattering energies, which is much larger than the present experimental and numerical uncertainties. We attribute this disagreement between the simulation and the experimental data to a breakdown of the IEM. The dynamic modification of the target-electron density leads to a change of the interaction potential (dressed projectile or dynamical screening). This effect goes beyond the IEM and has not been considered in this work. It leads to a reduction of the ionization/excitation probabilities and may be responsible for the observed differences.

Considering the small deviations between the final experimental and theoretical results in this work, however, one should apply the full Monte Carlo solution in order to reduce theoretical uncertainties to a minimum. Furthermore, it might be necessary to apply methods similar to time-dependent Hartree-Fock in order to resolve the current discrepancy between experimental data and theoretical results.

\section{CONCLUSIONS}

The line shape of the energy loss for $98 \mathrm{keV}$ protons on clean $\mathrm{Al}(110)$ was measured with high resolution and simulated using ab initio calculations of the energy-loss spectrum for single collisions as a function of the impact parameter. We have observed that large energy losses arising from inner-shell ( $L$-shell) ionization/excitation are responsible for the surface peak asymmetry. We note that the appropriate methods to handle the energy-loss line shape under shadowing/blocking conditions are those from the atomic physics field.

The long-term goal of our work is to establish the basic procedures and steps that are necessary to achieve clear and unequivocal monolayer resolution for solid-state materials analysis based on measured energy-loss spectra. However, even when using the most precise current methods of calculating the electronic energy loss (through the coupledchannel method AO) and appropriate slowing down statistics, small but significant disagreements are still observed between the experimental data and the simulation. These deviations are attributed to a breakdown of the independentelectron model. In this way, measurements of the energy loss under shadowing/blocking conditions might serve to im- 
prove our understanding of dynamically correlated electronic systems.

\section{ACKNOWLEDGMENTS}

This work was partially supported by the Brazilian agencies CNPq, CAPES, by the program for Brazilian-German cooperation PROBRAL 121/00 and by the US National Science Foundation under Grant No. DMR 0218406.

\section{APPENDIX A}

The hitting probability $p_{\text {hit }}$ is the chance to hit a certain target atom in a close encounter with asymptotically small impact parameters. It is normalized such that it equals unity in the case of an undisturbed flux of incident ions, as is the case for the topmost surface atoms. The incident projectile flight direction in front of the surface shall be given by the unit vector $\hat{e}_{i n}$. For an atom with index $i, p_{h i t}$ is given by

$$
p_{\text {hit }}^{(i)}=\int d^{3} \vec{l}_{i} F\left(\hat{e}_{i n}, \vec{r}_{i}+\vec{l}_{i}\right) G\left(\vec{l}_{i}, \sigma_{i}\right),
$$

where $F\left(\hat{e}_{i n}, \vec{r}\right)$ is the normalized flux of impinging ions ( $F=1$ for incident ions in front of the surface) that reach an atom located at the lattice position $\vec{r}_{i}+\vec{l}_{i}$. Here $\vec{r}_{i}$ is the mean lattice position and $\vec{l}_{i}$ is the thermal displacement with respect to the mean position with its time average $\left\langle\vec{l}_{i}\right\rangle_{t}=0$. If atom $i$ is located below the surface the flux at $\vec{r}_{i}+\vec{l}_{i}$ is reduced inside the shadow cones $(F \approx 0)$. $G(\vec{l}, \sigma)$ is a threedimensional Gaussian probability (normalized to unity) that represents the distribution of thermal atomic displacements (with 1D rms deviation $\sigma$ ). Thus, $p_{\text {hit }}$ defined in this way is one for the atoms on top of the surface (since the flux is undisturbed, $F=1$ ), but usually not for other atoms behind.

In order to account for the combined thermal vibrations of a surface atom and a bulk atom behind that atom in the beam direction $\left(\hat{e}_{i n}\right)$, we replace the flux $F$ (given in absolute spatial coordinates) by $\widetilde{F}$ given in spatial coordinates relative to a specific surface atom with index $k$. The effect of an individual shadow cone due to the $k_{t h}$ atom located at $\vec{r}_{k}+\vec{l}_{k}$ on the $i_{t h}$ target atom reads

$$
\begin{aligned}
p_{\text {hit }}^{(i, k)} & =\int d^{3} \vec{l}_{i} \widetilde{F}\left(\hat{e}_{i n}, \vec{r}_{i}+\vec{l}_{i}-\vec{r}_{k}-\vec{l}_{k}\right) G\left(\vec{l}_{i}-\vec{l}_{k}, \sqrt{\sigma_{i}^{2}+\sigma_{k}^{2}}\right) \\
& =\int d^{2} l_{k \perp} d l_{i \|} F\left(\hat{e}_{i n}, \vec{r}_{k}+\vec{l}_{k}\right) G\left(\vec{l}_{i}-\vec{l}_{k}, \sqrt{\sigma_{i}^{2}+\sigma_{k}^{2}}\right) \\
& =\int d^{2} b d l_{i \|} G\left(\vec{l}_{i}-\vec{l}_{k}, \sqrt{\sigma_{i}^{2}+\sigma_{k}^{2}}\right),
\end{aligned}
$$

where $\vec{l}_{i}=\vec{l}_{i \perp}+l_{i \|} \hat{e}_{i n}$ and $\vec{l}_{k}=\vec{l}_{k \perp}+l_{k \|} \hat{e}_{i n}$. In going from Eq. (A2) to Eq. (A3) we have used the ion-flux continuity equation $F\left(\hat{e}_{i n}, \vec{r}_{l}+\vec{l}_{i}\right) d^{2} l_{i \perp}=F\left(\hat{e}_{i n}, \vec{r}_{k}+\vec{l}_{k}\right) d^{2} l_{k \perp}$, where the relation between the areas $d^{2} l_{i \perp}$ and $d^{2} l_{k \perp}$ is given by the ion trajectory that connects the positions vectors $\vec{r}_{l}+\vec{l}_{i}$ and $\vec{r}_{k}+\vec{l}_{k}$. Note that $b$ is the impact parameter relative to the $k_{t h}$ atom ( $b=l_{k \perp}$ and $\left.d^{2} b=d^{2} l_{k \perp}\right)$ in Eq. (A4). Furthermore, since the ion flux at the $k_{t h}$ target atom $F\left(\hat{e}_{i} n, \vec{r}_{k}\right)$ is close to one (or equal to one for an atom at the surface), the flux does not appear explicitly in this equation. Equation (A4) can further be rewritten as

$$
p_{h i t}^{(i, k)}=\int d^{2} b g\left(\vec{l}_{i \perp}-\vec{l}_{k \perp}, \sqrt{\sigma_{i}^{2}+\sigma_{k}^{2}}\right)
$$

with

$$
g \equiv \int d l_{i \|} G\left(\vec{l}_{i}-\vec{l}_{k}, \sqrt{\sigma_{i}^{2}+\sigma_{k}^{2}}\right) .
$$

The transversal position $l_{i \perp}$ as a function of the impact parameter $b$ is calculated from

$$
l_{i \perp}=b+\left(r_{k \|}+l_{k \|}-r_{i \|}-l_{i \|}\right) \theta
$$

with the projectile-scattering angle $\theta$ calculated from the Moliere interatomic potential ${ }^{17}$ corresponding to atom $k$. Here we have approximated the projectile path by a piecewise straight-line trajectory considering only small-angle scattering $(\tan \theta=\theta)$.

The hitting probability for a given backscattering layer $i$ $=L$, including the combined action of all possible shadow cones that affect this layer, may approximately be written as

$$
p_{H}=\prod_{k}^{L} p_{h i t}^{(L, k)},
$$

using Eq. (A5). Of course, this procedure does not account correctly for overlapping shadow cones or correspondingly for multiple collision sequences with comparable deflections. For the present case however, all the involved approximations are expected to be of minor importance. This becomes obvious from the very close agreement of the present results with those from the well-accepted VEGAS Monte Carlo $\operatorname{program}^{3}$ as can be seen from Table I.

\section{APPENDIX B}

The angular deviation $\theta$ due to a shadow cone modifies slightly the incident angle and energy distribution of the backscattering collision. As discussed in the following, two effects can lead to such a modification. First, the Rutherford scattering cross section increases significantly for smaller scattering angles, enhances the backscattering contribution from one side of the shadow cone and gives rise to a corresponding shift of the backscattering peak. Second, the kinematical factor varies around the shadow cone (azimuthal direction), which will smear out the backscattering energy distribution. The change in the scattering angle will read

$$
\delta \Psi=\theta \sin (\phi),
$$

where $\phi$ is the azimuthal angle of the shadow cone. Under shadowing/blocking conditions the mean value of $\delta \Psi$ weighted with the Rutherford scattering cross section is very 
small for perfect crystals and projectiles in the MEIS energy regime. Thus, the angular spreading due to the shadow cone leads only to a small systematic energy shift of about $10 \mathrm{eV}$ that is neglected in the following. The same holds true for surface relaxation for the present conditions. However, the change of the scattering angle leads a change of the kinematical factor $K$ and to a related broadening of the energy distribution according to

$$
{\overline{\delta T^{2}}}_{i, k}=\left(\frac{\partial K}{\partial \Psi}\right)^{2} E_{0}^{2}{\overline{\delta \Psi^{2}}}_{i, k}
$$

with

$$
{\overline{\delta \Psi^{2}}}_{i, k}=\frac{1}{2}{\overline{\delta \theta^{2}}}_{i, k}
$$

and

*Deceased.

†Present address: Micron Technology, Inc., Process R\&D Center, 8000 S. Federal Way, Boise, ID 83707-006.

${ }^{1}$ G. de M. Azevedo, P.L. Grande, M. Behar, J.F. Dias, and G. Schiwietz, Phys. Rev. Lett. 86, 1482 (2001).

${ }^{2}$ J.F. van der Veen, Surf. Sci. Rep. 5, 199 (1985); R.M. Tromp, H.H. Kersten, E. Granneman, F.W. Saris, R. Koudijs, and W.J. Kilsdonk, Nucl. Instrum. Methods Phys. Res. B 4, 155 (1984).

${ }^{3}$ J.W.M. Frenken, R.M. Tromp, and J.F. van der Veen, Nucl. Instrum. Methods Phys. Res. B 17, 334 (1986).

${ }^{4}$ R.G. Smeenk, R.M. Tromp, H.H. Kersten, A.J.H. Boerboom, and F.W. Saris, Nucl. Instrum. Methods Phys. Res. 195, 581 (1982).

${ }^{5}$ R.M. Tromp, M. Copel, M.C. Reuter, M. Horn von Hoegen, J. Speidell, and R. Koudijs, Rev. Sci. Instrum. 62, 2679 (1991).

${ }^{6}$ H.H. Andersen, F. Besenbacher, P. Loftager, and W. Möller, Phys. Rev. A 21, 1891 (1980).

${ }^{7}$ W. Fritsch and C.D. Lin, Phys. Rep. 202, 1 (1991); J.F. Reading, T. Bronk, A.L. Ford, L.A. Wehrman, and K.A. Hall, J. Phys. B 30, L189 (1997).

${ }^{8}$ G. Schiwietz, Phys. Rev. A 42, 296 (1990); P.L. Grande and G. Schiwietz, 44, 2984 (1991); G. Schiwietz and P.L. Grande, Nucl. Instrum. Methods Phys. Res. B 69, 10 (1992); P.L. Grande and

$$
{\overline{\delta \theta^{2}}}_{i, k}=\int d^{2} b \theta(b)^{2} g\left(\vec{l}_{i \perp}-\vec{l}_{k \perp}, \sqrt{\sigma_{i}^{2}+\sigma_{k}^{2}}\right) .
$$

The factor $1 / 2$ in Eq. (B3) stems from the average over the angle $\phi$. Here $\vec{l}_{i}$ and $\vec{l}_{k}$ are the thermal or relaxation induced offsets of the positions of the backscattered atom and the target atoms that shadow and block the impinging ion. Thus the total additional energy spreading for a collision with a target atom from layer $L$ will read

$$
\overline{\delta E^{2}}=\left(\sum_{k=2}^{L} \overline{\delta T^{2}}{ }_{L, k}\right)_{\text {in }}+\left(\sum_{k=2}^{L} \overline{\delta T^{2}}\right)_{\text {out }} .
$$

The results from Eq. (7) have been convoluted with a Gaussian energy distribution with the above defined standard deviation (see Table I).
G. Schiwietz, Phys. Rev. A 47, 1119 (1993).

${ }^{9}$ J. Bang and J.M. Hansteen, Kgl. Dan. Vidensk. Selsk. Mat. Fys. Medd. 31, 13 (1959); L. Wilets and S.J. Wallace, Phys. Rev. 169, 84 (1968); M.R. Flannery and K.J. MacCann, Phys. Rev. A 8, 2915 (1973).

${ }^{10}$ G. Schiwietz and P.L. Grande, Current Applied Physics 3/1, 35 (2003).

${ }^{11}$ F. Herman and S. Skillmann, in Atomic Structure Calculations (Prentice-Hall, Englewood Cliffs, NJ, 1963).

${ }^{12} K=\left(\sqrt{m_{2}^{2}-m_{1}^{2} \sin ^{2} \Psi}+m_{1} \cos \Psi /\left(m_{1}+m_{2}\right)\right)^{2}$, where $m_{1}$ and $m_{2}$ are the projectile and target masses and $\Psi$ is the scattering angle in the laboratory system.

${ }^{13}$ J.R. Noonan and H.L. Davis, Phys. Rev. B 29, 4349 (1984).

${ }^{14}$ B.W. Busch and T. Gustafsson, Phys. Rev. B 61, 16097 (2000).

${ }^{15}$ D.S. Gemmel, Rev. Mod. Phys. 46, 129 (1974).

${ }^{16}$ Similar to the VEGAS Monte Carlo calculation (Ref. 3) but extended to take into account the inelastic energy losses. Further details will be described in a forthcoming publication.

${ }^{17}$ P. Gombas, in Statistische Behandlung des Atoms, edited by S. Fluegge, Handbuch der Physik (Springer-Verlag, Berlin, 1956), Vol. 36. 